\title{
Hyaluronic acid for repairing interdental papilla in esthetic area: case report.
}

\author{
Thaiz Zatta da Silva ${ }^{1 *}$, Rogério Margonar ${ }^{1}$, Rafael Silveira Faeda ${ }^{1}$, Aline de Oliveira ${ }^{1}$, Indira Cavalcanti de \\ Souza², Pâmela Letícia dos Santos ${ }^{1}$, Thallita Pereira Queiroz ${ }^{1}$.
}

1. Department of Health Sciences, Implantology Post Graduation Course, University of Araraquara UNIARA Dental School, SP, Brazil.

2. Piracicaba Dental School. State University of Campinas FOP-UNICAMP.

* Corresponding author: Thallita Pereira Queiroz. | Address : Rua Carlos Gomes, 1338, Centro - Araraquara-SP / Brasil | E-mail:thaqueiroz@ hotmail.com | Fone: +55 16 3301-7408

Work received on 23/03/2019.

Approved for publication on 11/08/2019.

\begin{abstract}
Background: Hyaluronic acid is a treatment option for this deficiency gingival papilla, since it aims to increase tissue volume in the region of loss of interdental papilla, minimally invasive. The aim this study was to describe the application of Hyaluronic acid in a deficient gingival papilla. Case report: Patient presented absence of interdental papilla in the maxillary arch around upper anterior teeth. A comprehensive exam was performed, plus 2D photographs taken with a professional camera coupled to a device for image standardization, and an intraoral scanning with the OMNICAM scanner. Three applications of Hyaluronic acid were performed to fill the black spaces, at an interval of four weeks. The patient was followed-up for three months, and the recovery of papilla was observed. Conclusion: The Hyaluronic acid as gingival filling material can be considered as a treatment option for augmenting interdental papilla in aesthetic area. KEY WORDS:
\end{abstract}

Interdental papilla; Hyaluronic acid; CAD/CAM; Dental technology

Rev. Clin. Periodoncia Implantol. Rehabil. Oral Vol. 12(3); 157-158, 2019

\section{INTRODUCTION}

The interdental papilla is classified as a non-keratinized or parakeratinized gingival tissue covered by stratified squamous (flattened) epithelium, and clinically occupies the physiological space between anterior teeth with a pyramidal shape ${ }^{(1)}$. It does not only act as biological barrier to protect periodontal structures but also play a fundamental role in esthetics.

Several factors may lead to the loss of interdental papilla, including loss of teeth and bone resorption in the area of alveolar ridge associated with periodontal disease, in which the distance from the point of contact should be greater than or equal to $5 \mathrm{~mm}$ in natural teeth ${ }^{(2)}$. The treatments are proposed for interdental papilla reconstruction, as connective tissue graft, acellular dermal matrix, surgical flaps and guided tissue regeneration (GTR) therapy ${ }^{(3)}$. However, these procedures are still unpredictable and have a high morbidity rate, in addition to require high surgical precision and presenting risks of clinical complications ${ }^{(4)}$. Recently, the hyaluronic acid $(\mathrm{HA})$ has been indicated for reconstruction of interdental papilla in the cervical region of teeth and crowns aiming to repair or improve defects with positive results ${ }^{(5)}$.

Based on the above we are reporting the use of HA in esthetic area aiming to increase the volume of gingival tissue and to promote formation of papilla in black spaces. The clinical results and follow-up of this case showed that HA can be recommended for non-surgical treatment of cases of black triangle defects.

\section{CASE DESCRIPTION}

A healthy 51-year-old female patient came to the oral care with chief complain being "a dark space between front teeth that bothers me", which was characterized as a vertical defect of papilla. The medical history did not reveal any systemic impairment that could affect the healing if any treatment was proposed. The clinical exam charted an internal hex implant in the area of tooth number 2.1 (3.5 x 15 mm; Conexão, Arujá, Brazil) with immediate loading and in temporary restoration phase.

The defective papillaes were located between elements 1.1 and 2.1, and between 2.1 and 2.2. During the periodontal probing more than $3 \mathrm{~mm}$ was found around the area but no bleeding and no mobility of the implant, suggesting health. Based on the clinical condition it was proposed a minimally invasive approach as treatment for filling the space of the interdental papilla with application of HA prior to the final prosthodontic treatment. The patient was advised about possible risks, complications and benefits of the treatment proposed, but agreed and signed a free and informed consent form.

The initial conditions were recorded by photographs using a Canon T3i macro lens camera, supported on a photographic device for image standardization. An oral scan with OMNICAM Sirona camera (Dentsply Sirona CAD / CAM - CEREC Omnicam Dental CAD / CAM System, York, United States) was also performed in order to obtain a three-dimensional evaluation of the case (Fig. 1A, 1B and 1C).

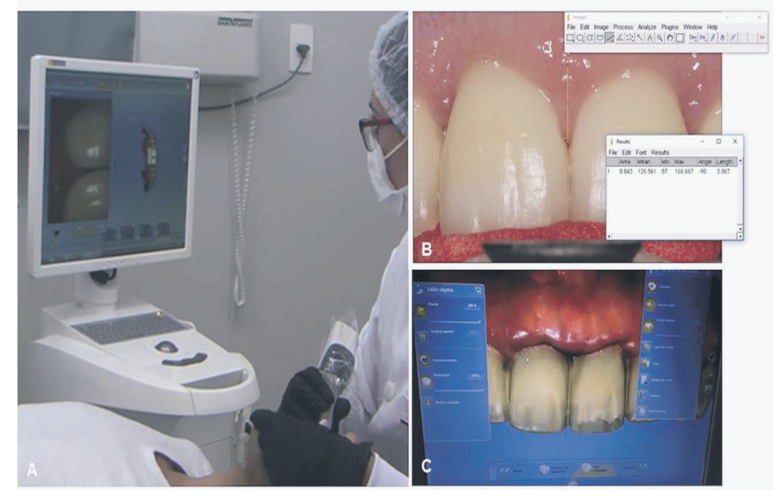

Figure 1: (A) Intra oral scanning with OMNICAM Sirona camera (Dentsply Sirona CAD / CAM - CEREC Omnicam Dental CAD / CAM System, York, United States) (B) obtaining the measure and (C) 3D evaluation of the case.

Due to the use of this device the images have been taken at the same angulations and distance from the patient's face. This step was important for comparison and follow-up. The images were taken (and labelled) as follow in Figure 2: T0 = Initial (Fig. 2A); T1 = immediately after application (Fig. 2B); T2 $=1$ month after the application (Fig. 2C); T3 = 2 months after the application (Fig. 2D) and T4 $=3$ months after the application (Fig. 3).

For all HA application, a previous prophylaxis and a numbing with topical anesthetic Xylestesin Spray 10 \% (CRISTÁLIA, Itapira, Brazil) was performed on the gum tissue that will receive the HA gel. We applied a volume between 0.1 and $0.2 \mathrm{~mL}$ which corresponded to $23 \mathrm{mg} / \mathrm{ml}$ of HA Rennova Fill gel (Innovapharma, São Paulo, Brazil), $3 \mathrm{~mm}$ apical to the tip of papilla with a 27 G1/2 needle until extravasation of material via gingival sulcus ${ }^{(5-6)}$. The application was then performed three more times, with an interval of four weeks between applications. The patient was followed up throughout the treatment.

After three months of follow-up, a pink-colored healthy gingiva was 


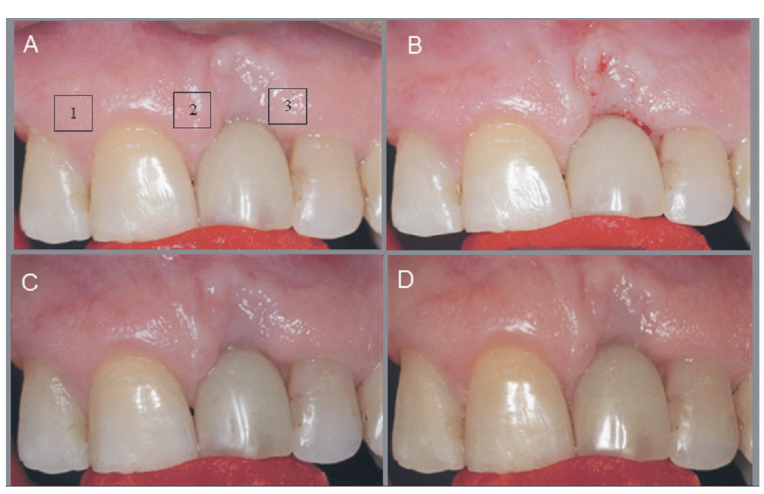

Figure 2: Images obtained with aid of photographic device for $(A)$ T0 $=$ Initial; (B) T1 = immediately after application; (C) T2 = 1 month after the application and (D) T3 $=2$ months after the application.

observed and the papilla with a satisfactory tissue filling. The black space between teeth was reduced and softened. The use of AH did not cause any clinical morbidity to the patient (Fig.3.)

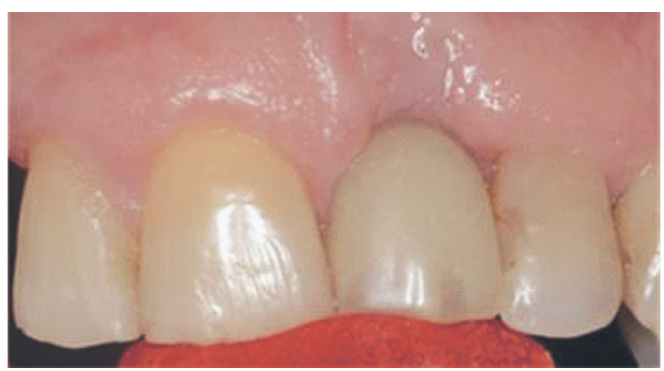

Figure 3: Final image 3 months after the application (T4).

\section{DISCUSSION}

This clinical report evaluated the effect of HA application on tissue augmentation of papilla in esthetic area. The patient was followed for three months after the first application of $\mathrm{HA}$, and was possible to suggest the use of $\mathrm{HA}$ as a non-surgical treatment option for black space recovery. The treatment is safe, effective and has a low rate of morbidity for the patient, which agreed with other studies published in the literature ${ }^{(7-9)}$. The papilla that received the gel applications had an extensive tissue defect around the implant. The interdental papilla is a structure with minor blood supply and was left untouched by clinicians due to sometimes unpredictable outcomes, letting the reconstruction of interdental papilla to be one of the most challenges in periodontal treatment. We can suggest that the success of the treatment was due to the treatment plan, to the procedure itself and importantly to the HA characteristic as high solubility within the gum tissue which provided volume, sustentation and elasticity to the tissues.

Several clinical studies have evaluated non-animal originated HA as a type of treatment for the esthetic reconstruction of the interdental papilla in the anterior teeth ${ }^{(7-9)}$. We observed satisfactory results after four months of application while other authors suggested recovery of papilla after six months. These authors evaluated more patients and used a different gel brand. The patients had at least one area of the interdental papilla presenting class I or II defects, but the applications were performed using the same volume of gel as we did on the base of the papilla. The injection was repeated two more times after 21 days and all patients were checked monthly. They showed improvement in papilla around 0.6 to $1.2 \mathrm{~mm}^{(7)}$.

Lee and colleagues (2016) ${ }^{(8)}$ used HA application in interdental papilla of esthetic area, and evaluated its reconstruction with radiographies and anatomical factors. Fifty-seven sites from 13 patients (6 males and 7 females) who had papillae deficiency in the upper anterior area were included in their study. As in this study, these authors used photographic devices to standardize images. In this context, our study also highlights the importance of using devices for image standardization. These authors used a device to inject the HA gel into the papilla. The applications were repeated up to five times every three weeks. Patients were followed for six months after the initial application. The measurements of black triangle area (BTA), size (BTS) and width (BTW), periapical radiographic measurements of the contact point and bone crest (CP - $B C)$ and interproximal distance between roots (IDR) were performed by computer. Interdental papillary reconstruction rate (IPRR) was calculated to determine the gain in BTA and reconstruction of the interdental papilla. All sites showed improvement and reconstruction of the interdental. The CP-BC suggested full reconstruction of papilla.

Although several cases reports have been published in the literature, there are still not many protocols for using HA on gum tissue reconstruction. For this reason, $\mathrm{Pi}$ and colleagues $(2017)^{(9)}$ developed a protocol based on the techniques used by several professionals for treatment of black spaces. They developed an animal model with papillae defect mimicking a black space and later performed the HA applications. After the applications the shape of papilla was more regular and smoother than the initial condition. The local application of $\mathrm{AH}$ was validated as a significant and minimally invasive procedure to improve the filling.

In the present paper, the volumetric analysis of the interdental papillae was performed by the intraoral three-dimensional scanning with Sirona CAD I CAM, being measured by overlap using the equipment software itself. It is believed that the distortion of the measures in these cases is low because it is a high technology equipment. Through these analyzes it was possible to note that HA gel applications promoted gain of interdental papilla tissue.

\section{CONCLUSION}

Within the limitations of this clinical report we suggest that HA gel applications promoted gain of interdental papilla tissue and can be recommended as a treatment option for recovery of pink tissue volume that directly compromises esthetic. It is minimally invasive and can safely be used.

\section{CLINICAL RELEVANCE}

The deficient gingival papilla compromises soft tissue esthetics, mainly in patients submitted to prosthetic rehabilitation and orthodontic treatment. Hyaluronic acid is a treatment option for this deficiency, since it aims to increase tissue volume in the region of loss of interdental papilla, minimally invasive. It is a promising therapy with a favorable diagnosis, but needs further scientific evidence.

\section{CONFLICT OF INTEREST}

The authors do not have any financial interest in the companies whose materials are included in this article.

\section{ACKNOWLEDGMENTS}

The authors would like to acknowledge Innovapharma S.A, Rennova, Goiânia, GO, Brazil, for supporting the treatment reported in this paper.

\section{REFERENCES}

1. Choen B. Morphological factors in the pathogenesis of the periodontal disease. Br Dent J. 1959;7:31-9.

2. Tarnow DP, Magner AW, Fletcher P. The effect of the distance from the contact point to the crest of bone on the presence or absence of the interproximal dental papila. J Periodontol. 1992;63(12):995-6.

3. Giannobile WV. Commentary: Treatment of periodontitis: destroyed periodontal tissues can be regenerated under certain conditions. J Periodontol. 2014;85(9):1151-4 4. Anitua E, Troya M, Orive G. Plasma rich in growth factors promote gingival tissue regeneration by stimulating fibroblast proliferation and migration and by blocking transforming growth factor-beta1-induced myodifferentiation. J Periodontol. 2012;83(8):1028-37.

5. Hunt DR, Jovanovic SA, Wikesjo UM, et al. Hyaluronan supports recombinant human bone morphogenetic protein-2 induced bone reconstruction of advanced alveolar ridge defects in dogs. A pilot study. J Periodontol. 2001;72(5):651-8.
6. Becker W, Gabitov I, Stepanov M, et al. Minimally invasive treatment for papillae deficiencies in the esthetic zone: a pilot study. Clin Implant Dent Relat Res. 2010;2(1):1-8

7. Sadat MS, Ghasemi M, Salmani Z, et al. Clinical application of hyaluronic acid gel for reconstruction ofInterdental papilla at the esthetic zone. J Islam Dent Ass IRAN 2013;25(2):191-6.

8. Fatin A, Awartani, Tatakis DN. Interdental papilla loss: treatment by hyaluronic acid gel injection: a case series. Clin Oral Invest. 2016;20:1775-80.

9. Lee WP, Kim HJ, Yu SJ, et al. Six month clinical evaluation of interdental papilla reconstruction with injectable hyaluronic acid gel using an image analysis system. J Esthet Restor Dent. 2016;28:221-30.

10. Pi S, Choi YJ, Hwang $\mathrm{S}$, et al. Local injection of hyaluronic acid filler improves open gingival embrasure: validation through a rat model. J Periodontol. 2017;88(11):1221-30. 